OPEN ACCESS

Edited by:

Massimo Fantini,

Precision Biologics, Inc., United States

Reviewed by: Lasse Dahl Ejby Jensen, Linköping University, Sweden Andrea Sartore-Bianchi, University of Milan, Italy

${ }^{*}$ Correspondence: Xin-Hua Zhu xzhu1@northwell.edu

${ }^{t}$ These authors have contributed equally to this work

Specialty section: This article was submitted to Cancer Molecular Targets and Therapeutics, a section of the journal Frontiers in Oncology

Received: 27 January 2021 Accepted: 28 July 2021 Published: 31 August 2021

Citation:

Quinn R, Patel R, Sison C, Singh A and Zhu X-H (2021) Impact of Precision Medicine on Clinical Outcomes: A Single-Institution Retrospective Study.

Front. Oncol. 11:659113

doi: 10.3389/fonc.2021.659113

\section{Impact of Precision Medicine on Clinical Outcomes: A Single- Institution Retrospective Study}

\author{
Ryann Quinn ${ }^{1 \dagger}$, Rajvi Patel ${ }^{1 \dagger}$, Cristina Sison ${ }^{2,3}$, Amandeep Singh ${ }^{1}$ and Xin-Hua Zhu ${ }^{1,3 *}$ \\ ${ }^{1}$ Northwell Health Cancer Institute, New Hyde Park, NY, United States, ${ }^{2}$ Biostatistics Unit, Feinstein Institutes for Medical \\ Research, Northwell Health, Manhasset, NY, United States, ${ }^{3}$ Donald and Barbara Zucker School of Medicine at Hofstral \\ Northwell, Hempstead, NY, United States
}

Purpose: The strategy of precision medicine has been widely adopted in the practice of oncology, although the efficacy remains unclear. This study assesses clinical outcomes in patients with an actionable alteration found during FoundationOne $\mathrm{CDx}^{\mathrm{TM}}(\mathrm{F} 1 \mathrm{CDx})$ testing and who received a targeted therapy based on the results.

Materials and Methods: This is a retrospective cohort study of patients with tumors that underwent F1CDx from September 2012 to July 2018. F1CDx provided actionable alterations for patients to select appropriate therapies. The primary objective was to estimate the objective response rate (ORR) at 3 months from the start of study treatment. The secondary objectives were to estimate progression-free survival (PFS) and overall survival (OS).

Results: One thousand patients underwent F1CDx testing. Six hundred fifty-two patients were identified as having actionable mutations. Thirty-eight patients (18 males and 20 females) received targeted therapy and were included in the study. The most common alterations were PD-1/PDL-1, high-TMB, P13K, and HER2/ERBB2. Patients received various treatments including nivolumab, pembrolizumab, trastuzumab, and everolimus. Eight (23.5\%) and six (17.7\%) patients achieved partial response (PR) and stable disease (SD), respectively; 20 (58.8\%) had progression of disease (PD). The disease control rate was $41.2 \%$ (95\% Cl: $24.7 \%$ to 59.3\%). The median PFS was 2.7 months (95\% Cl: 2.3 to 5.4 months), and median OS was 9.9 months (95\% Cl: 4.5 to 33.7 months).

Conclusion: Our results demonstrate promising data in precision medicine in real community oncology practice. It warrants further large and prospective studies in patients with actionable alterations.

Keywords: precision medicine, FoundationOne, next-generation sequencing, precision oncology, targeted treatment approaches 


\section{INTRODUCTION}

Advances in genomics and next-generation sequencing (NGS) have revolutionized the field of oncology by allowing precisionbased approaches in management of cancer. Precision oncology looks at genetic and molecular characteristics of tumors instead of traditional histology to match treatment strategies (1). This strategy has been applied in the management of many types of cancers including testing epidermal growth factor receptor (EGFR) mutation, anaplastic lymphoma kinase (ALK) fusion, Btype Raf kinase (BRAF) mutation, c-Ros oncogene 1 (ROS1), and programmed death ligand-1 (PD-L1) in nonsmall-cell lung cancer (NSCLC); human epidermal growth factor receptor 2 (HER2) in breast and gastric cancer; Kirsten rat sarcoma viral oncogene homolog (K-RAS), neuroblastoma ras viral oncogene homolog (N-RAS), and BRAF mutation in colon cancer; and tumor mutational burden, NTRK fusions, and microsatellite instability (MSI) status in all solid tumors (2-8). Biomarker-matched targeted therapies for specific alterations in approximately 40 different cancer genes are available that are Food and Drug Administration (FDA) approved $(9,10)$. In addition, many precision clinical trials in oncology which aim to determine if targetable gene alterations can predict response to targeted therapies were done and are currently being conducted. Results from selected arms of the National Cancer Institute-Molecular Analysis for Therapy Choice (NCI-MATCH) trial are available and have mixed results. While the BRAF V600E/K mutation cohort (dabrafenib and trametinib) and the MSI-H cohort (nivolumab) had positive results, other arms such as the fibroblast growth factor receptor (FGFR) alteration cohort (FGFR inhibitor AZD4547), the PIK3CA-mutation cohort (taselisib), and the HER2 amplification cohort (ado-trastuzumab emtansine) had negative results (11-15). The biomarkers PDL-1 and tumor mutation burden (TMB) have been shown to predict responses to immunotherapy in several cancers and are also included in our study as "targeted treatment".

The efficacy of precision oncology remains unclear. However, the strategy of precision medicine has been widely adopted in the practice of community oncology. The FoundationOne $\mathrm{CDx}{ }^{\mathrm{TM}}$ test $\left(\mathrm{F} 1 \mathrm{CDx}{ }^{\mathrm{TM}}\right)$ was the first NGS-based gene panel to be approved by the FDA for the detection of alterations that may confer benefit from FDA-approved treatments for certain cancers (16). Two additional panels (Memorial Sloan Kettering (MSK)-IMPACT and Oncomine Dx Target Test) have subsequently been approved to detect tumor gene alterations in certain cancer types (17). At our institution, we frequently order F1CDx testing on patients with advanced cancer resistant to standard of care, or in rare cancers in which there is no standard of care. It is necessary to report the efficacy and problems such as low treatment rate of precision medicine in the real world among patients with multiple targetable alterations and multiple cancer types. We conducted a retrospective cohort study of patients treated at our institution that received nonstandard-of-care-targeted therapy based on the results of F1CDx testing. This study examines our experience with clinical outcomes in patients identified as having an actionable alteration through $\mathrm{F} 1 \mathrm{CDx}$ testing and who received a targeted therapy based on the results.

\section{MATERIALS AND METHODS}

We performed a retrospective cohort study of patients with tumors that underwent F1CDx from September 2012 to July 2018 at the Northwell Health Monter Cancer Center. The study was approved by our institutional review board. Patients were identified using the Foundation Medicine patient reports database. Patients were excluded if they were less than 18 years of age or if F1CDx testing was done for a hematologic malignancy. Patients' age, diagnosis, ethnicity, number of prior treatments, actionable alterations identified by F1CDx testing, and available targeted treatments were recorded. We also calculated the growth modulation index (GMI) for each patient that had a prior line of therapy by calculating the ratio of progression-free survival (PFS) on targeted therapy $\left(\mathrm{PFS}_{n}\right)$ vs. PFS on prior line of treatment (PFSn-1).We used the electronic medical record to determine if patients received the targeted treatment suggested by the F1CDx report; if they did not receive targeted treatment, we recorded the next treatment received after F1CDx testing if available. We excluded patients who received targeted therapy that was FDA approved and standard of care for the patient's primary tumor site. Of note, patients with MSI-H and tumor mutational burden included in this study received targeted treatments prior to FDA approval dates.

\section{Statistical Considerations}

The primary objective of the study was to estimate the objective response rate (ORR) at 3 months from the start of study treatment. ORR was defined by imaging findings as per Response Evaluation Criteria in Solid Tumors version 1.1 (RECIST v1.1) (18). Secondary objectives were to estimate PFS and overall survival (OS).

Descriptive statistics (frequencies, proportions, means, standard deviations, medians, IQR) were calculated to describe the demographic and clinical characteristics. The ORR at 3 months from start of treatment was estimated using standard methods for estimating proportions and their associated 95\% confidence intervals. The Kaplan-Meier product limit method was used to estimate PFS and OS. PFS was measured from the time of initiation of targeted treatment therapy to the first documentation of disease progression or death due to any cause. Patients who have not achieved the event of interest (progression or death for PFS and death for OS) as of the last documented follow-up were considered "censored" and the last follow-up time was used in the analysis.

\section{Sample Size Considerations}

While there were 652 patients with actionable mutations in the F1CDx database, only 38 patients (5.8\%) received targeted therapy based on the results and were included in the study. Thus, the total sample size for this study was 38 subjects. The sample size was a sample size of convenience and was 
based on feasibility and availability of subjects that meet inclusion criteria; it was not based on any formal statistical power calculations.

\section{RESULTS}

One thousand patients at our institution had tumor samples sent for F1CDx testing from September 2012 to July 2018. Six hundred fifty-two patients had actionable alterations with available targeted treatments. Of the 652 patients, 42 (6.4\%) went on a clinical trial, 165 (25\%) received standard next-line chemotherapy, 135 (20.7\%) received targeted therapy, 144 either went on hospice or died prior to receiving treatment (22\%), 142 were lost to follow-up (22\%), 21 were treated with surgery only (3.2\%), and three $(0.04 \%)$ had issues with insurance approval of targeted therapy (Figure 1). Of the 135 patients that were treated with targeted therapy, 97 received FDA approved targeted therapy (15\%) and 38 (5.7\%) received non-FDA-approved targeted therapy. The 38 patients that received non-FDAapproved targeted therapy were included in the analysis.

There were a total of 38 patients ( 18 males and 20 females) in the study sample (Table 1). Mean age of patients was 57.7 years, ranging from 20 to 92 years. The majority $(35 / 38=92.1 \%)$ had at least one other prior systemic therapy. Patients included in the study had tumors with various targetable alterations (Figure 2). The most common alterations were the PD-1/PDL-1 (eight patients, $21.1 \%$ ) and high-TMB (eight patients, $21.1 \%$ ). There were four patients with $\mathrm{P} 13 \mathrm{~K}$ gene $(10.5 \%)$ and four patients with HER2/ERBB2 (10.5\%). Patients were diagnosed with various types of primary tumors including breast, gastrointestinal, head and neck, lung, neuroendocrine, ovarian, urothelial, and unknown primary adenocarcinoma (Table 2). There were nine patients with colon adenocarcinoma, five patients with unknown primary adenocarcinoma, and three patients with urothelial cell carcinoma. Patients received
TABLE 1 | Patient characteristics.

\begin{tabular}{lc}
\hline Characteristic & No. of patients (\%) \\
\hline Sex & \\
Female & $20(53 \%)$ \\
Male & $18(47 \%)$ \\
Median age [years (range)] & $58(20-92)$ \\
Race/ethnicity & \\
White & $21(55 \%)$ \\
Black & $10(26 \%)$ \\
Asian & $4(11 \%)$ \\
Hispanic & $2(5 \%)$ \\
Declined & $1(3 \%)$ \\
Prior lines of therapy) \\
0 & $3(8 \%)$ \\
1 & $8(21 \%)$ \\
2 & $10(26 \%)$ \\
3 & $11(29 \%)$ \\
$>3$ & $6(16 \%)$
\end{tabular}

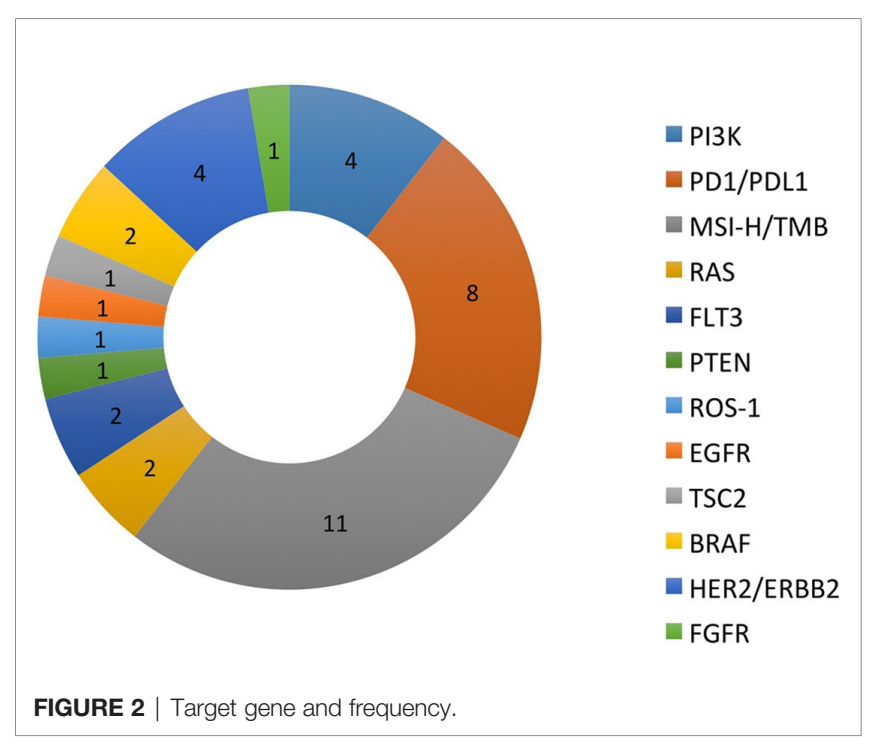

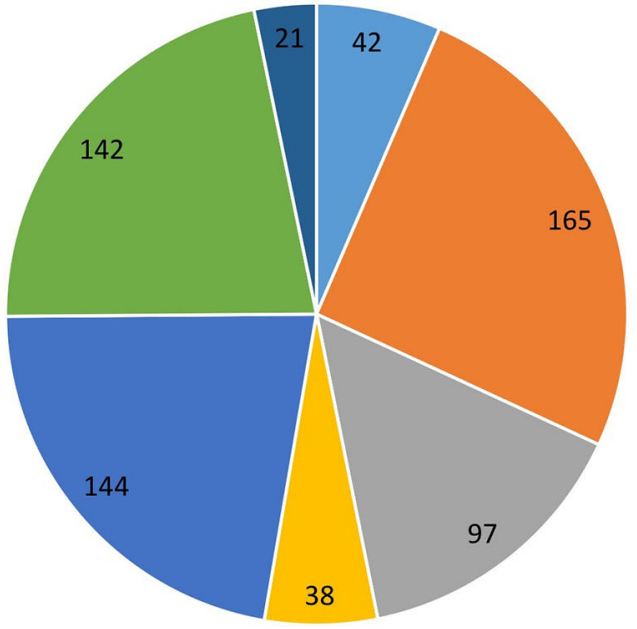

FIGURE 1 | Treatment received after results of F1CDx testing available.
Clinical Trial

- Standard Chemotherapy

FDA Approved Targeted Treatment

Non-FDA approved targeted treatment

- Hospice

Lost to follow up

- Surgery only 
TABLE 2 | Tumor histology.

\begin{tabular}{lc}
\hline Tumor histology & Frequency \\
\hline Breast & 1 \\
Breast invasive ductal carcinoma & 1 \\
Breast angiosarcoma & \\
Gastrointestinal & 9 \\
Colon adenocarcinoma & 7 \\
Other gastrointestinal adenocarcinoma & \\
Lung & 1 \\
Lung adenocarcinoma & 1 \\
Genitourinary & 3 \\
Kidney epitheliod angiomyolipoma & 1 \\
Urothelial cell carcinoma & 1 \\
Gynecologic & 1 \\
Cervical squamous cell carcinoma & 1 \\
Ovarian adenocarcinoma & 1 \\
Uterine carcinosarcoma & 1 \\
Head and neck & 1 \\
Squamous cell carcinoma & \\
Thymic carcinoma & 1 \\
Neuroendocrine & \\
Neuroendocrine carcinoma & 1 \\
Sqin & \\
Unknownown primary adenocarcinoma & 1 \\
\hline
\end{tabular}

various targeted treatments including nivolumab (nine patients; 23.7\%), pembrolizumab (eight patients; $21.1 \%$ ), trastuzumab (four patients; 10.5\%), and everolimus (four patients; 10.5\%); treatment distribution of the rest of the 13 patients is shown in Figure 3. The molecular alterations and matched treatment for each patient is listed in Table 3. The average time from F1CDx testing to receiving targeted treatment was 184 days. The median time was 97 days.

Of the 1,000 total patients tested, the most common alteration was KRAS (200 patients, 20\%), followed by PI3K gene alterations
(65 patients, 6.5\%) and BRAF alterations (60 patients, 6\%). Prevalence of the 15 most common gene alterations found in $>10$ patients is shown in Table 4.

Of the 38 patients, $34(89.5 \%)$ had a first interval scan performed from 1 to 5 months after start of targeted therapy; 13 of the 38 patients $(34.2 \%)$ had a second interval scan performed between 4 to 9 months after initiation of targeted treatment therapy; and seven of the 38 patients $(18.4 \%)$ had a third interval scan performed between 10 and 16 months after the start of targeted treatment therapy. Follow-up times ranged from 0.8 to 16.1 months. The median follow-up time was 2.7 months (mean, 5.1 months, $\mathrm{SD}=4.3$ months).

Of the 34 patients evaluated for their ORR at the first scan, there were eight $(23.5 \%)$ and six (17.7\%) with PR and SD, respectively; 20 (58.8\%) had PD. Of the 13 patients with available data at the second scan, nine (69.2\%) and three (23.1\%) had PR and SD, respectively; one (7.7\%) had PD. Of the seven patients with available data at the third scan, one (14.3\%), three (42.9\%), and two (28.6\%) had CR, PR, and SD, respectively; one (14.3\%) had PD (Figure 4). Note that for the second and third interval scans, the number of evaluable observations decreased substantially due to patients having progression of disease or death after the first interval scan or not yet reaching the timepoint for the 2 nd or 3 rd interval scans. The interpretation of the proportions of ORR and favorable response rates for the second and third interval scans should be interpreted with caution due to potential missing information.

The median overall survival was estimated to be 9.9 months (95\% CI: 4.5 to 33.7 months) (Figure 5). Neither gender or age at diagnosis was associated with overall survival. The median timeto-progression was estimated to be 2.7 months (95\% CI: 2.3 to 5.4 months) (Figure 6). Neither gender or age at diagnosis was associated with progression-free survival.

Nine (27\%) out of 33 patients who were eligible for PFS ratio analysis demonstrated a PFS ratio of $\geq 1.3$ with exact binomial of $95 \%$ and confidence interval of $13 \%$ to $46 \%$ (Table 5).

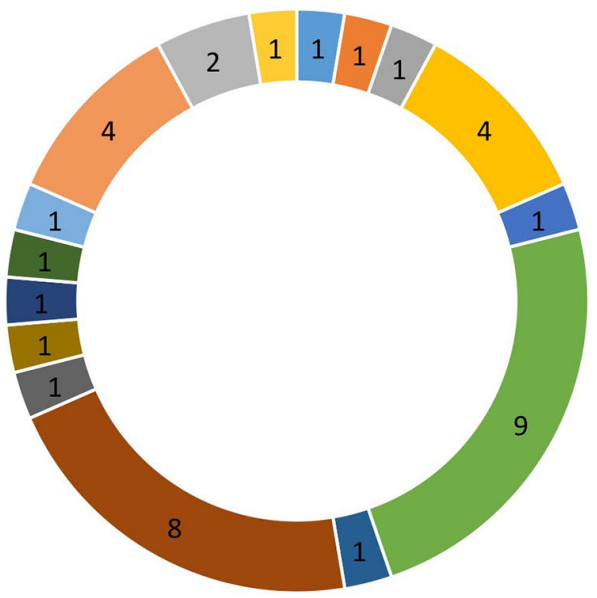

- Atezolizumab
Crizotinib
Erlotinib
Everolimus
- Ipilimumab/Nivolumab
- Nivolumab
- Pazopanib
- Pembrolizumab
- Ponatanib
Regorafenib
- Sorafenib
- Temsirolimus
- Trametenib/Dabrafenib
Trastuzumab
- Tremetinib

FIGURE 3 | Targeted treatment received and frequency. 
TABLE 3 | Molecular alteration and matched therapy.

\begin{tabular}{|c|c|c|c|}
\hline Patient & Diagnosis & Molecular alteration & Matched therapy \\
\hline 1 & Urothelial cell carcinoma & PI3K & Temsirolimus \\
\hline 2 & Colon adenocarcinoma & High TMB & Pembrolizumab \\
\hline 3 & Colon adenocarcinoma & High TMB & Nivolumab \\
\hline 4 & Esophageal adenocarcinoma & PTEN & Everolimus \\
\hline 5 & Small intestine adenocarcinoma & ROS-1 & Crizotinib \\
\hline 6 & Colon adenocarcinoma & MSI-H & Pembrolizumab \\
\hline 7 & Neuroendocrine carcinoma (rectal) & PDL-1 & Nivolumab \\
\hline 8 & Unknown primary adenocarcinoma & PDL-1 & Nivolumab \\
\hline 9 & Cervix-squamous cell carcinoma & PI3K & Everolimus \\
\hline 10 & Skin squamous cell carcinoma & MSI-H & Nivolumab \\
\hline 11 & Duodenal adenocarcinoma & MSI-H & Nivolumab \\
\hline 12 & Head and neck squamous cell carcinoma & RAS & Trametinib \\
\hline 13 & Unknown primary adenocarcinoma & EGFR & Erlotinib \\
\hline 14 & Neuroendocrine lung carcinoma & PDL-1 & Nivolumab \\
\hline 15 & Kidney epithelioid angiomyolipoma & TSC2 & Everolimus \\
\hline 16 & Colon adeocarcinoma & FLT3 & Ponatanib \\
\hline 17 & Lung adenocarcinoma & BRAF & Vemurafenib \\
\hline 18 & Colon adenocarcinoma & RAS & Tremetinib \\
\hline 19 & Breast angiosarcoma & $\mathrm{PI3K}$ & Sorafenib \\
\hline 20 & Colon adenocarcinoma & HER2/ERBB2 & Trastuzumab \\
\hline 21 & Unknown primary adenocarcinoma & HER2/ERBB2 & Trastuzumab \\
\hline 22 & Thymic carcinoma & PDL-1 & Pembrolizumab \\
\hline 23 & Unknown primary adenocarcinoma & PDL-1 & Nivolumab \\
\hline 24 & Rectal adenocarcinoma & High TMB & Pembrolizumab \\
\hline 25 & NET with small cell features & High TMB & Ipilimumab/Nivolumab \\
\hline 26 & Urothelial carcinoma & PI3K & Everolimus \\
\hline 27 & Esophageal adenosquamous carcinoma & High TMB & Pembrolizumab \\
\hline 28 & Breast cancer & High TMB & Pembrolizumab \\
\hline 29 & Pancreatic adenocarcinoma & HER2/ERBB2 & Trastuzumab \\
\hline 30 & Colon adenocarcinoma & HER2/ERBB2 & Trastuzumab \\
\hline 31 & Ovarian cancer & PDL-1 & Pembrolizumab \\
\hline 32 & Uterine carcinosarcoma & FGFR & Pazopanib \\
\hline 33 & Gastric adenocarcinoma & PDL-1 & Pembrolizumab \\
\hline 34 & Unknown primary squamous cell carcinoma & High TMB & Nivolumab \\
\hline 35 & Urothelial carcinoma & High TMB & Atezolizumab \\
\hline 36 & Unknown primary adenocarcinoma & PDL-1 & Nivolumab \\
\hline 37 & Colon adenocarcinoma & FLT3 & Regorafenib \\
\hline 38 & Colon adenocarcinoma & BRAF & Trametenib/Dabrafenib \\
\hline
\end{tabular}

TABLE 4 | Prevalence of actionable alterations among 1,000 patients studied.

\begin{tabular}{lcc}
\hline Alteration & Number of patients & Percentage (\%) \\
\hline KRAS & 200 & 20 \\
PIK3CA & 65 & 6.5 \\
BRAF & 60 & 6 \\
TMB & 57 & 5.7 \\
ERBB2/HER2 & 55 & 5.5 \\
STK11 & 52 & 5.2 \\
EGFR & 32 & 3.2 \\
PDL-1 & 31 & 3.1 \\
FGFR1 & 29 & 2.9 \\
FLT3 & 26 & 2.6 \\
CCND1 & 25 & 2.5 \\
BRCA2 & 18 & 1.8 \\
NF2 & 16 & 1.6 \\
KIT & 16 & 1.6 \\
PDGFRA & 11 & 1.1
\end{tabular}

We did not carry out a formal statistical test since we did not prespecify a hypothesis regarding the proportion of subjects with a $P F S \geq 1.3$.

\section{DISCUSSION}

In this study, eight of 34 (23.5\%) patients who had a 3-month interval scan had a partial response. Of these eight patients, three had a PDL-1 overexpression, two had a BRAF mutation, and three had sustained PR for $>1$ year; these patients all received immunotherapy for microsatellite instability (MSI-high). Two patients received nivolumab and one patient received pembrolizumab. One patient had a sustained CR for $>1$ year; this patient had lung adenocarcinoma and received vemurafenib for a BRAF mutation. Six (17.6\%) patients showed stable disease. Disease control rate including partial response and stable disease was $41.1 \%$. Even if we remove the three cases of MSI-high patients based on current standard of care, five of $31(16.1 \%)$ with partial response and six of 31 with stable disease (19.3\%), thus disease control rate was $35.4 \%$. Although our study included multiple targetable genes and multiple cancer types which reflects the real practice of precision medicine in community oncology, and was retrospective, the objective response rate was greater than $16 \%$ in either the above-mentioned analysis. 
25

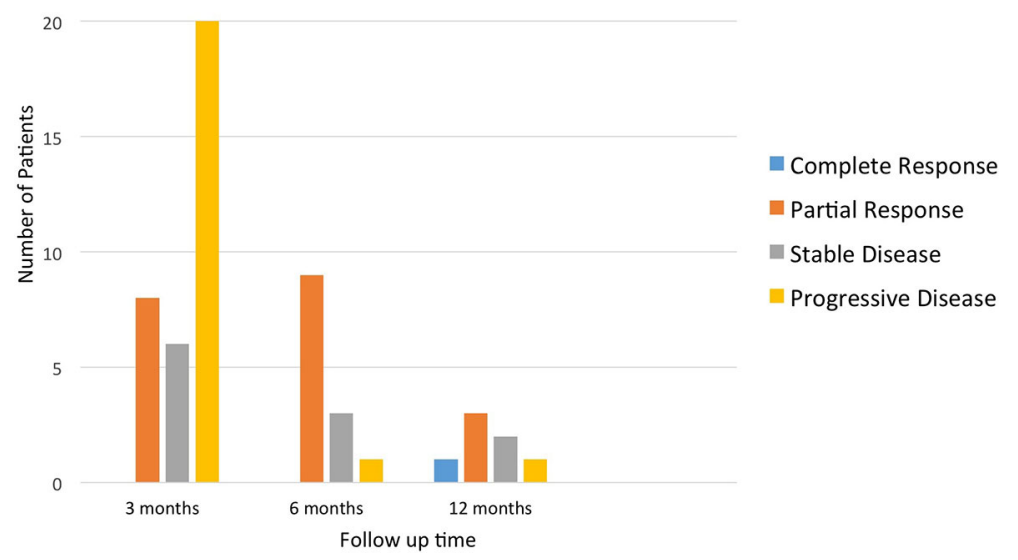

FIGURE 4 | Overall response rate of patients who received targeted treatment at different time points.

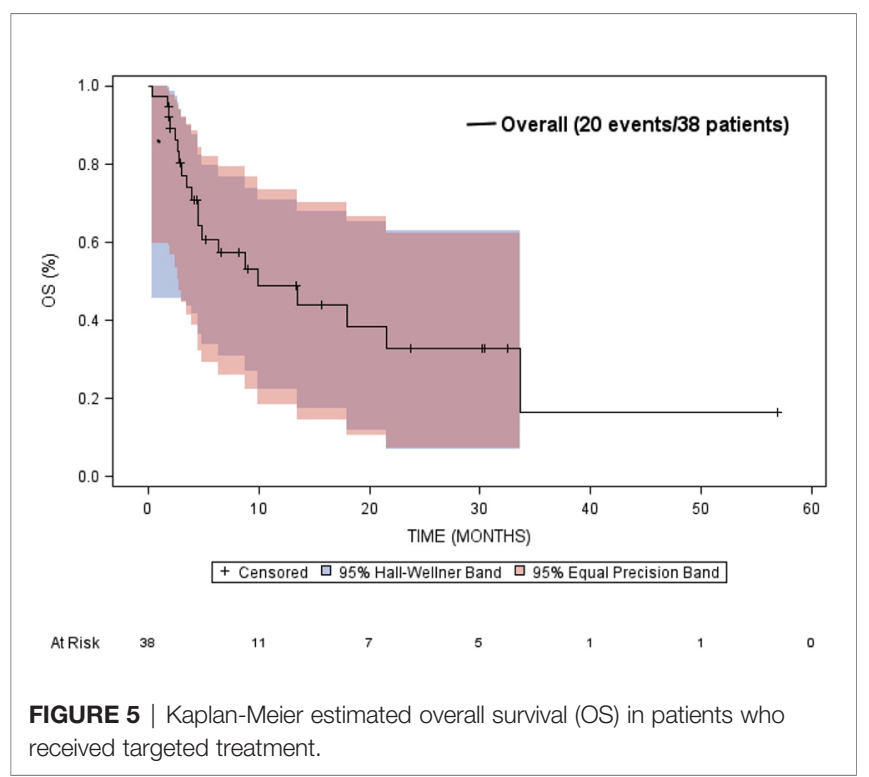

This threshold was set as a promising tumor shrinkage in the NCI-MATCH trial.

In this study, 1,000 patients had F1CDx testing and 652 patients had tumors that had actionable alterations. Of these 652 patients, $42(6.4 \%)$ went on a clinical trial, 135 (20.7\%) received targeted therapy, 97 received FDA-approved targeted therapy $(15 \%)$, and $38(5.8 \%)$ received non-FDA-approved targeted therapy. The low rate of patients receiving targeted therapy even in the presence of actionable alterations has also been seen in other studies (19-24). In the MOSCATO-1 trial, of the 411 tumors which were found to have potentially actionable alterations, only $199(48 \%)$ were paired with a

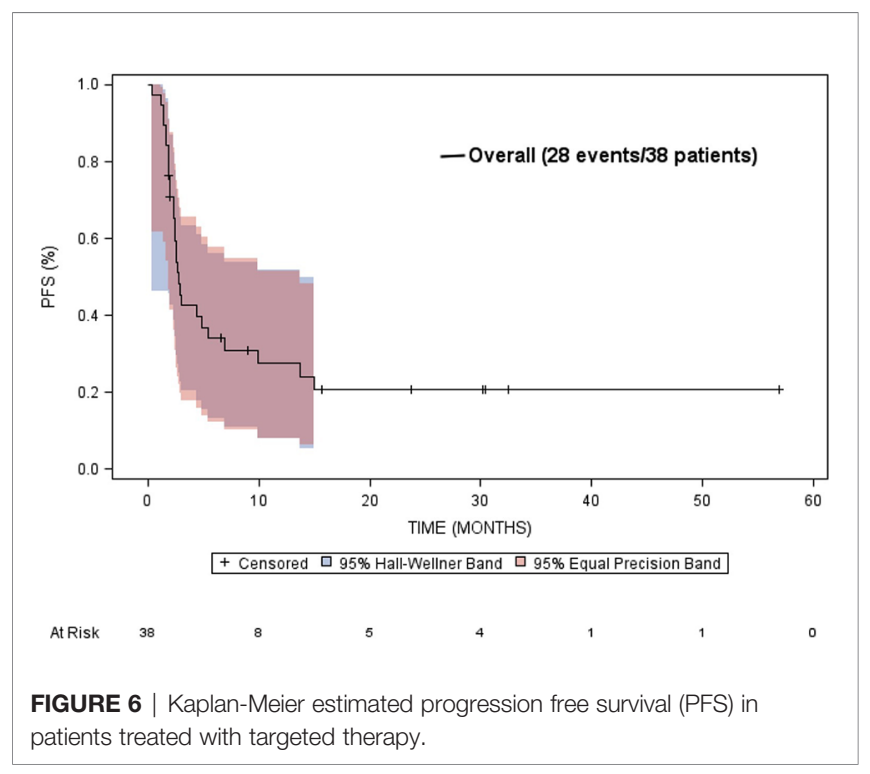

targeted therapy (19). The patients on these studies had similar reasons for not receiving targeted treatment, such as, rapid clinical deterioration, enrolling on a different clinical trial, or being lost to follow-up. At our center, as well as in other trials, a major factor preventing patients from receiving targeted therapy was decline in performance status as well as patients electing hospice rather than further therapy. This highlights the importance of shared decision making, which takes a patient's values, performance status, and time to testing results prior to requesting NGS testing. On the other hand, one challenge with multigene NGS sequencing is that providers may have a challenge selecting the "best option" when multiple 
TABLE 5 | Treatment regimen received by nine patients with ratio of PFS $\geq 1.3$.

\begin{tabular}{|c|c|c|c|}
\hline Patient & Diagnosis & Previous regimen prior to target treatment & Target treatment received \\
\hline 6 & Colon adenocarcinoma & Fluorouracil + oxaliplatin & Pembrolizumab \\
\hline 7 & Neuroendocrine carcinoma (rectal) & Capecitabine + oxaliplatin & Nivolumab \\
\hline 8 & Unknown primary adenocarcinoma & Fluorouracil + oxaliplatin & Nivolumab \\
\hline 11 & Duodenal adenocarcinoma & Fluorouracil + oxaliplatin & Nivolumab \\
\hline 14 & Neuroendocrine lung carcinoma & Carboplatin + etoposide & Nivolumab \\
\hline 17 & Lung adenocarcinoma & Carboplatin + pemetrexate & Vemurafenib \\
\hline 18 & Colon adenocarcinoma & Irinotecan + regorafenib & Trametinib \\
\hline 26 & Urothelial carcinoma & Atezolizumab & Everolimus \\
\hline 38 & Colon adenocarcinoma & Regorafenib & Trametinib/Dabrafenib \\
\hline
\end{tabular}

targetable drugs are available for an actionable alteration. F1CDx reported many variants of uncertain significance or equivocal copy number alterations, further complicating the selection. A recent study at an academic medical center showed that $22 \%$ of physicians reported low confidence in their knowledge of genetics (25). One study of 5,688 patients with advanced NSCLC who were treated in the community setting showed that of the 873 patients who received broad panel NGS testing, less than $5 \%$ of patients received nonapproved targeted treatment based on the results (26). The development of regional molecular tumor boards including geneticists and bioinformaticists may provide improved identification of potential off-label or investigational therapeutic options as well as assist with navigation of insurance authorization (27). Our center does not have a molecular tumor board which could have contributed to the low treatment rate. There was low enrollment of 42 patients $(6.4 \%)$ on precision medicine trials.

Twenty-five percent of patients at our center who had F1CDx testing done received the next-line standard-of-care treatment. Given that broad NGS testing is unlikely to affect first-line treatment, oncologists should consider waiting until the patient relapses or is refractory to standard-of-care treatments before sending a tumor sample for NGS testing. The genetic makeup of a patient's tumor frequently changes during the course of the disease due to intratumor heterogeneity and is often responsible for development of resistance to chemotherapy or targeted therapy. Therefore, it may be more prudent to wait until the patient progresses through standard-of-care therapies prior to sending F1CDx. This could have caused the poor response rate we saw in our study, as many of our patients were treated with targeted therapy several months or years after F1CDx testing was done. Another consideration is that current Medicare reimbursement guidelines state that Medicare will only pay for F1CDx one time per each malignancy, so the treating physician will be unable to send a repeat F1CDx panel at a later date without a large cost to the patient (28). In our study, the average time between when F1CDx testing was done and when patients received targeted treatment was 184 days, the median time was 97 days. Therefore, many patients had another line of treatment in between when the testing was done and when they received targeted treatment. It is possible that the genetic makeup of the tumor could have changed during that period.
Limitations of the study include that it was a single-center retrospective study and the small sample size. Given the small sample size, multiple different targetable alterations and tumor types were included and analyzed together, which limits the ability to analyze the efficacy of each individual targetable alteration. Given the 2.7 months median PFS, a significant proportion of patients progressed after the first scan. Larger prospective randomized controlled trials which focus on an individual gene alteration tested across multiple tumor types are needed to see if targeted therapies can improve response rates.

The GMI was developed by Von Hoff as a way to objectively evaluate PFS in studies with no control arm and in which there is no consensual standard of care treatment that can be used for comparison (29). It compares the time to progression on the study drug (PFSn) with the PFS with the prior line of therapy $\left(\mathrm{PFS} n_{-1}\right)$, and the ratio $(\mathrm{PFS} n) /\left(\mathrm{PFS}_{-1}\right)$ is calculated, therefore using each patient as their own control. Using the assumption that PFS becomes shorter with each successive line of treatment, a conservative ratio of $>1.3$ has been used to signify drug efficacy. In our study, nine of 33 patients (27\%) achieved this ratio. Given that our study was a single-arm trial with multiple different targeted treatments used and no control group, we thought this could be a reasonable measure of efficacy.

We found $23.5 \%$ patients with partial response rate, $17.6 \%$ patients with stable disease. Disease control rate was $41.1 \%$ for the patients who received targeted treatment based on results of F1CDx testing. Our results demonstrate promising data in precision medicine in real-community oncology practice. It warrants further large and prospective studies in patients with actionable alterations. Given the low treatment rate, the experience at our cancer center recommends patient selection with good performance status and proper timing for submitting NGS. There is high demand for high-quality regional molecular tumor boards to improve the selection of targetable gene alterations. Making more oncologists aware of precision medicine trials will also help improve patients' treatment rate. In our study, 1,000 patients received F1CDx testing and only 14 patients (1.4\%) received benefit from targeted treatment. Larger studies are needed to identify the proportion of patients that will benefit from precision medicine, especially given the high cost of the testing. The use of precision medicine is rapidly increasing, our future direction is to include more patients so that we can analyze actionable alterations separately, similar to the NCI-MATCH trial. 


\section{DATA AVAILABILITY STATEMENT}

The raw data supporting the conclusions of this article will be made available by the authors, without undue reservation.

\section{ETHICS STATEMENT}

The studies involving human participants were reviewed and approved by Northwell Health IRB. Written informed

\section{REFERENCES}

1. Park JJH, Hsu G, Siden EG, Thorlund K, Mills EJ. An Overview of Precision Oncology Basket and Umbrella Trials for Clinicians. CA Cancer J Clin (2020) 70(2):125-37. doi: 10.3322/caac.21600

2. Lindeman NI, Cagle PT, Aisner DL, Arcila ME, Beasley MB, Bernicker EH, et al. Updated Molecular Testing Guideline for the Selection of Lung Cancer Patients for Treatment With Targeted Tyrosine Kinase Inhibitors: Guideline From the College of American Pathologists, the International Association for the Study of Lung Cancer, and the Association for Molecular Pathology. J Mol Diagn (2018) 20(2):129-59. doi: 10.1016/j.jmoldx.2017.11.004

3. Bartley AN, Washington MK, Colasacco C, Ventura CB, Ismaila N, Benson $\mathrm{AB} 3 \mathrm{rd}$, et al. HER2 Testing and Clinical Decision Making in Gastroesophageal Adenocarcinoma: Guideline From the College of American Pathologists, American Society for Clinical Pathology, and the American Society of Clinical Oncology. J Clin Oncol (2017) 35(4):446-64. doi: 10.1200/JCO.2016.69.4836

4. Benson AB III, Venook AP, Al-Hawary MM. NCCN Guidelines for Colon Cancer. Version 2 (2018). (Accessed June 28, 2018).

5. Marabelle A, Le DT, Ascierto PA, Di Giacomo AM, De Jesus-Acosta A, Delord JP, et al. Efficacy of Pembrolizumab in Patients With Noncolorectal High Microsatellite Instability/Mismatch Repair-Deficient Cancer: Results From the Phase II KEYNOTE-158 Study. J Clin Oncol (2020) 38(1):1-10. doi: 10.1200/JCO.19.02105

6. Hong DS, DuBois SG, Kummar S, Farago AF, Albert CM, Rohrberg KS, et al. Larotrectinib in Patients With TRK Fusion-Positive Solid Tumours: A Pooled Analysis of Three Phase 1/2 Clinical Trials. Lancet Oncol (2020) 21:531. doi: 10.1016/S1470-2045(19)30856-3

7. Demetri GD, Paz-Ares L, Farago AF, Liu SV, Chawla SP, Tosi D, et al. Efficacy and Safety of Entrectinib in Patients With NTRK Fusion-Positive (NTRK-Fp) Tumors: Pooled Analysis of STARTRK-2, STARTRK-1, and ALKA-372-001. Ann Oncol (2018) 29:viii713. doi: 10.1093/annonc/mdy424.017

8. Marcus L, Lemery SJ, Keegan P, Pazdur R. FDA Approval Summary: Pembrolizumab for the Treatment of Microsatellite Instability-High Solid Tumors. Clin Cancer Res (2019) 25:3753-8. doi: 10.1158/1078-0432.CCR-184070

9. Kumar-Sinha C, Chinnaiyan AM. Precision Oncology in the Age of Integrative Genomics. Nat Biotechnol (2018) 36(1):46-60. doi: 10.1038/ nbt. 4017

10. Malone ER, Oliva M, Sabatini PJB, Stockley TL, Siu LL. Molecular Profiling for Precision Cancer Therapies. Genome Med (2020) 12(1):8. doi: 10.1186/ s13073-019-0703-1

11. Salama AKS, Li S, Macrae ER, Park J-I, Mitchell EP, Zwiebel JA, et al. Dabrafenib and Trametinib in Patients with Tumors with BRAF V600E Mutations: Results of the NCI-MATCH Trial Subprotocol H. J Clin Oncol (2020) 6:JCO2000762. doi: 10.1200/JCO.20.00762

12. Azad NS, Gray RJ, Overman MJ, Shoenfeld JD, Mitchell EP, Zwiebel JA, et al. Nivolumab Is Effective in Mismatch Repair-Deficient Noncolorectal Cancers: Results From Arm Z1D-A Subprotocol of the NCI-MATCH (EAY131) Study. J Clin Oncol (2020) 38(3):214-22. doi: 10.1200/JCO.19.00818

13. Chae YK, Hong F, Vaklavas C, Cheng HH, Hammerman P, Mitchell EP, et al. Phase II Study of AZD4547 in Patients With Tumors Harboring Aberrations in the FGFR Pathway: Results From the NCI-MATCH Trial (EAY131) Subprotocol W. J Clin Oncol (2020) 38(21):2407-17. doi: 10.1200/JCO.19.02630 consent for participation was not required for this study in accordance with the national legislation and the institutional requirements.

\section{AUTHOR CONTRIBUTIONS}

All authors listed have made a substantial, direct, and intellectual contribution to the work and approved it for publication.

14. Krop IE, Jegede O, Grilley-Olson E, Lauring JD, Hamilton SR, Zwiebel JA, et al. Results From Molecular Analysis for Therapy Choice (MATCH) Arm I: Taselisib for PIK3CA-Mutated Tumors [Abstract]. J Clin Oncol (2018) 36(15 suppl):101. doi: 10.1200/JCO.2018.36.15_suppl.101

15. Jhaveri KL, Wang XV, Makker V, Luoh SW, Mitchell EP, Zwiebel JA, et al. Ado-Trastuzumab Emtansine (T-DM1) in Patients With HER2-Amplified Tumors Excluding Breast and Gastric/Gastroesophageal Junction (GEJ) Adenocarcinomas: Results From the NCI-MATCH Trial (EAY131) Subprotocol Q. Ann Oncol (2019) 30(11):1821-30. doi: 10.1093/annonc/ mdz291

16. PMA P170019: FDA Summary of Safety and Effectiveness Data. FDA

17. Zeng J, Johnson A, Shufean MA, Kahle M, Yang D, Woodman SE, et al. Operationalization of Next-Generation Sequencing and Decision Support for Precision Oncology. JCO Clin Cancer Inform (2019) 3:1-12. doi: 10.1200/ CCI.19.00089

18. Eisenhauer EA, Therasse P, Bogaerts J, Schwartz LH, Sargent D, Ford R, et al. New Response Evaluation Criteria in Solid Tumours: Revised RECIST Guideline (Version 1.1). Eur J Cancer (2009) 45:228-47. doi: 10.1016/ j.ejca.2008.10.026

19. Massard C, Michiels S, Ferté C, Le Deley MC, Lacroix L, Hollebecque A, et al. High-Throughput Genomics and Clinical Outcome in Hard-to-Treat Advanced Cancers: Results of the MOSCATO 01 Trial. Cancer Discovery (2017) 7(6):586-95. doi: 10.1158/2159-8290.CD-16-1396

20. Sohal DPS, Khorana BI, Dreicer AA, Abraham R, Procop J, Saunthararajah GW, et al. Prospective Clinical Study of Precision Oncology in Solid Tumors. J Natl Cancer. Inst (2015) 108(3):djv332. doi: 10.1200/jco.2015.33.15_suppl.6585

21. Meric-Bernstam F, Brusco L, Shaw K, Horombe C, Kopetz S, Davies MA, et al. Feasibility of Large-Scale Genomic Testing to Facilitate Enrollment Onto Genomically Matched Clinical Trials. J Clin Oncol (2015) 33:2753-62. doi: 10.1200/JCO.2014.60.4165

22. André F, Bachelot T, Commo F, Campone M, Arnedos M, Dieras V, et al. Comparative Genomic Hybridisation Array and DNA Sequencing to Direct Treatment of Metastatic Breast Cancer: A Multicentre, Prospective Trial (SAFIR01/UNICANCER). Lancet Oncol (2014) 15:267-74. doi: 10.1016/ S1470-2045(13)70611-9

23. Beltran H, Eng K, Mosquera JM, Sigaras A, Romanel A, Rennert H, et al. Whole-Exome Sequencing of Metastatic Cancer and Biomarkers of Treatment Response. JAMA Oncol (2015) 1:466-74. doi: 10.1001/jamaoncol.2015.1313

24. Stockley TL, Oza AM, Berman HK, Leighl NB, Knox JJ, Shepherd FA, et al. Molecular Profiling of Advanced Solid Tumors and Patient Outcomes With Genotype-Matched Clinical Trials: The Princess Margaret IMPACT/ COMPACT Trial. Genome Med (2016) 8(1):109. doi: 10.1186/s13073-0160364-2

25. Gray SW, Hicks-Courant K, Cronin A, Rollins BJ, Weeks JC. Physicians' Attitudes About Multiplex Tumor Genomic Testing. J Clin Oncol (2014) 32:1317-23. doi: 10.1200/JCO.2013.52.4298

26. Presley CJ, Tang D, Soulos PR, Chiang AC, Longtine JA, Adelson KB, et al. Association of Broad-Based Genomic Sequencing With Survival Among Patients With Advanced Non-Small Cell Lung Cancer in the Community Oncology Setting. JAMA (2018) 320:469-77. doi: 10.1001/jama.2018.9824

27. Knepper TC, Bell GC, Hicks JK, Padron E, Teer JK, Vo TT, et al. Key Lessons Learned From Moffitt's Molecular Tumor Board: The Clinical Genomics Action Committee Experience. Oncologist (2017) 22:144-51. doi: 10.1634/ theoncologist.2016-0195 
28. Decision Memo for Next Generation Sequencing (NGS) for Medicare Beneficiaries With Advanced Cancer (CAG-00450N). In Database MC (Ed.). Center of Medicare \& Medicaid Services. Available at: https://www.cms.gov/ medicare-coverage-database/details/nca-decision-memo.aspx?NCAId= 290\&bc $=$ AAAAAAAAACAA\& (Accessed June 20, 2020).

29. Von Hoff DD, Stephenson JJJr, Rosen P, Loesch DM, Borad MJ, Anthony S, et al. Pilot Study Using Molecular Profiling of Patients' Tumors to Find Potential Targets and Select Treatments for Their Refractory Cancers. J Clin Oncol (2010) 28(33):4877-83. doi: 10.1200/JCO.2009.26.5983

Conflict of Interest: The authors declare that the research was conducted in the absence of any commercial or financial relationships that could be construed as a potential conflict of interest.
Publisher's Note: All claims expressed in this article are solely those of the authors and do not necessarily represent those of their affiliated organizations, or those of the publisher, the editors and the reviewers. Any product that may be evaluated in this article, or claim that may be made by its manufacturer, is not guaranteed or endorsed by the publisher.

Copyright (๑) 2021 Quinn, Patel, Sison, Singh and Zhu. This is an open-access article distributed under the terms of the Creative Commons Attribution License (CC BY). The use, distribution or reproduction in other forums is permitted, provided the original author(s) and the copyright owner $(s)$ are credited and that the original publication in this journal is cited, in accordance with accepted academic practice. No use, distribution or reproduction is permitted which does not comply with these terms. 